\title{
Revisão do Stent Coronário com Eluição de Sirolimus
}

\author{
Alexandre Abizaid ${ }^{1}$, Fabricio Ribeiro Las Casas¹, José Fábio Almiro da Silva
}

\section{RESUMO}

O stent coronário com eluição de sirolimus foi aprovado pela CE Mark, na Europa, em abril de 2002, e nos Estados Unidos, pelo Food and Drug Administration (FDA), em abril de 2003. Desde os resultados iniciais do estudo de viabilidade First In Man (FIM), alguns trabalhos randomizados, controlados, documentaram o poder antiproliferativo do sirolimus, um antibiótico macrolídeo e potente inibidor citostático da proliferação de células musculares lisas. O benefício clínico aumentou após a segunda fase de estudos envolvendo lesões mais complexas (como reestenose intrastent, vasos pequenos, oclusões crônicas) e pacientes de alto risco, como os diabéticos. Recentemente, foi possível comparar os dois stents com liberação de fármacos comercialmente disponíveis nos Estados Unidos, em cinco estudos do tipo "cabeça a cabeça". Com o resultado de vários estudos multicêntricos e unicêntricos, nacionais e internacionais, em que a segurança e a eficácia do stent coronário com eluição de sirolimus foram avaliadas de forma minuciosa, hoje, a comunidade global de Cardiologia Intervencionista tem evidências para apoiar o uso dessa tecnologia, resultando em significativa melhora dos desfechos nos pacientes submetidos a intervenção coronária percutânea.

DESCRITORES: Angioplastia transluminal percutânea coronária. Reestenose coronária. Contenedores. Sirolimus.

E 1979, quando Andreas Grüntzig publicou os achados dos primeiros 50 pacientes tratados com angioplastia, poucos poderiam prever o crescimento notável das intervenções coronárias percutâneas, associado à explosão da pesquisa clínica e à expansão do conhecimento adquirido. Não há dúvidas de que, após a introdução da angioplastia com balão, em 197711, o stent intracoronário foi a mais importante evolução no campo da revascularização coronária percutânea. No entanto, o problema da reestenose, meses após o procedimento inicial, continuou sendo um desafio aos cardiologistas intervencionistas. As conse-

\footnotetext{
1 Instituto Dante Pazzanese de Cardiologia - São Paulo, SP. Correspondência: Alexandre Abizaid. Setor de Angioplastia. Av. Dr. Dante Pazzanese, 500 - São Paulo, SP - CEP 04012-180 - Tel.: (11) 5085-6141 - Fax: (11) 5549-7807 • E-mail: aabizaid@uol.com.br Recebido em: 7/8/2007 - Aceito em: 24/10/2007
}

\section{SUMMARY}

Review of Sirolumus-Eluting Coronary Stents

The sirolimus-eluting coronary stent received CE Mark approval in Europe in April 2002. In the US, FDA approval followed in April 2003. Since the preliminary results from the First-in-Man feasibility study were presented, several randomized, controlled trials have documented the profound antiproliferative effects of sirolimus, a macrolide antibiotic and potent cytostatic inhibitor of smooth muscle cell proliferation. Subsequently, the body of clinical evidence was increased by the second wave of evidence from trials in more complex lesions (such as in-stent reestenosis, small vessels, chronic total occlusions) and "high-risk" patients such as those with diabetes. More recently we have had the opportunity to compare the two commercially available drug-eluting stents following the presentation of data from five head-to-head trials. As a result of numerous single and multi-center, national and international studies in which the safety and efficacy of sirolimus-eluting coronary stents have been subjected to close scrutiny, the global intervention cardiology community now has evidence in support of the use of this technology resulting in dramatically improved patient outcomes after percutaneous intervention.

DESCRIPTORS: Angioplasty, transluminal, percutaneous coronary. Coronary restenosis. Stents. Sirolimus.

qüências da reestenose são várias, afetando a imagem da angioplastia como terapêutica intervencionista definitiva. A recorrência dos sintomas tem impacto desfavorável para o paciente e para o sistema de saúde. Nova intervenção percutânea, necessidade de cirurgia de revascularização, reinternação e infarto do miocárdio levam a um impacto na qualidade de vida do paciente e da família, gerando custos adicionais que sobrecarregam a economia do sistema de saúde.

Em 2003, foi estimada a realização de mais de dois milhões de angioplastias em todo o mundo, resultando em cerca de 250 mil casos de reestenose clínica, dos quais aproximadamente $50 \%$ correspondem a reestenose recorrente (Tabela 1 ).

\section{Fisiopatologia da reestenose}

A reestenose após o implante do stent envolve uma complexa relação de eventos biológicos. Hoje, 
sabemos que o implante de stent causa não só injúria endotelial como injúria profunda à parede arterial. Essa injúria pode estimular a migração e a proliferação excessiva das células musculares lisas, com formação da matriz extracelular na região subendotelial². A presença de reestenose nas bordas do stent é principalmente causada pela injúria local do balão, que leva ao remodelamento negativo do vaso, e não pelo crescimento neointimal ${ }^{3}$.

Os stents sem eluição de fármacos não impedem a proliferação de células musculares lisas e o crescimento tecidual, o que faz da associação com fármacos o dispositivo mais promissor na luta contra a reestenose, na atualidade.

\section{O que é o sirolimus?}

O sirolimus foi isolado, pela primeira vez, de um microorganismo do solo, Streptomyces hygroscopicus, encontrado na Ilha de Páscoa, como descrito por Sehgal et al., em 19754. Rapa Nui é o nome local da Ilha de Páscoa, inspirando o nome bem conhecido, rapamicina.

O sirolimus cristalizado foi purificado com fermentação, tornando-se ativo contra vários filamentos fermentados de fungos e algumas bactérias ${ }^{4}$, levando à classificação original do sirolimus como um antibiótico antifúngico ${ }^{5}$.

Por ser pouco solúvel em água (lipofílico), quase não há liberação do fármaco na corrente sanguínea durante o implante do stent na lesão; após seu implante, o gradiente de difusão favorece a penetração no tecido, novamente limitando a circulação livre do sirolimus.

Em adição a essa atividade antibiótica, evidenciouse que o sirolimus também possui efeito antiproliferativo potente e propriedades imunossupressoras ${ }^{6}$. O sirolimus se mostrou um inibidor da proliferação celular, em diversos modelos in vitro e in vivo, de uma forma diferente da ciclosporina $\mathrm{A}$. As mesmas propriedades antiproliferativas de células musculares lisas foram confirmadas em numerosos modelos vasculares ${ }^{7}$.

Estudos in vivo e modelos de angioplastia demons-

TABELA 1

Reestenose: extensão do problema

\begin{tabular}{lc}
$\begin{array}{lc}\text { Intervenções coronárias } \\
\text { no mundo (2003) }\end{array}$ & $\begin{array}{l}\mathbf{2 , 0} \text { milhões }(\sim \mathbf{5 0} \% \\
\text { nos Estados Unidos })\end{array}$ \\
\hline Reestenose angiográfica: & $40 \%-45 \%$ \\
ATC & $15 \%-25 \%-350.000$ por ano \\
Stents & 240.000 por ano \\
Eventos clínicos & 120.000 por ano \\
Reestenose recorrente & \\
\hline ATC = angioplastia transluminal coronária.
\end{tabular}

traram a eficácia do sirolimus na prevenção de hiperplasia tecidual causada pela injúria vascular, levandonos a considerá-lo como agente na prevenção da reestenose.

\section{Estudos clínicos}

O estudo de viabilidade First In Man $(\mathrm{FIM})^{8}$ demonstrou notável eficácia em eliminar a ocorrência de reestenose com o uso do sirolimus. Esses resultados iniciais foram seguidos de achados sem precedentes do estudo RAVEL ${ }^{9}$, o primeiro estudo duplo-cego, randomizado e controlado usando o stent com liberação de sirolimus. Esses resultados são bem conhecidos e foram reproduzidos em três estudos controlados adicionais (SIRIUS, E-SIRIUS, e C-SIRIUS) $)^{10-12}$.

Desde os resultados iniciais do estudo de viabilidade FIM $^{8}$, o stent CYPHER está disponível em cerca de 80 países, sendo utilizado por mais de 1,5 milhão de pacientes em todo o mundo. Hoje, o stent com eluição de sirolimus é o mais estudado e apresenta maior evidência clínica, demonstrando segurança e eficácia do fármaco e do polímero a longo prazo. Sua eficácia se traduz nas significativas menores taxas de perda tardia, de reestenose tanto clínica como angiográfica e de eventos cardíacos maiores, principalmente à custa da menor revascularização do vasoalvo. Além disso, o stent com eluição de sirolimus demonstrou resultados convincentes nos desfechos de pacientes em que foi usada ou não a pré-dilatação com cateter-balão, de acordo com o estudo DIRECT ${ }^{13}$.

No entanto, a maior crítica de qualquer estudo clínico controlado é a limitação na seleção dos pacientes, mediada por rigorosos critérios de inclusão e de exclusão. Contra esse contexto é que alguns registros prospectivos foram iniciados, com o intuito de conseguir o maior número de dados em lesões mais desafiadoras (vasos pequenos, lesões longas, bifurcações, oclusões crônicas e lesão no tronco de coronária esquerda) e em pacientes mais complexos (como portadores de síndrome coronariana aguda e multiarteriais) (Tabela 2).

\section{Conduta em lesões complexas e pacientes de alto risco}

A experiência do operador com os stents farmacológicos cresce assim como as evidências clínicas demonstradas nos desfechos de uma série de estudos independentes, comparativos, do tipo "cabeça a cabeça", sendo usados em lesões progressivamente mais complexas e em pacientes considerados de alto risco.

Dentre os fatores associados a essa mudança da Cardiologia Intervencionista, três se destacam entre os demais:

1. a prevalência crescente de diabetes melito e de lesões mais complexas (vasos pequenos, doença mais extensa e difusa, oclusões totais, lesões do tronco de coronária esquerda); 
TABELA 2

Stent CYPHER - registros de vigilância pós-marketing

\begin{tabular}{lccc}
\hline Registro & País & Pacientes & Centros \\
\hline e-CYPHER & Internacional & 15.500 & 367 \\
BRIDGE & França & 1.000 & 100 \\
SCORPIUS & Alemanha & 190 & 19 \\
CYPHER-SMART & Itália & 256 & 19 \\
EVASTENT & França & 2.000 & 47 \\
DESSERT & Itália & 700 & 40 \\
CYPHER & Alemanha & 7.000 & 85 \\
\hline
\end{tabular}

2. a crescente epidemia de obesidade associada à síndrome metabólica e de aterosclerose precoce;

3. o advento de stents com liberação de fármacos, que resultou em melhores desfechos, levando a maior espectro de indicações (incluindo diabetes melito, multiarteriais, reestenose intra-stent, oclusões crônicas, bifurcações, enxertos de safena e lesões no tronco de coronária esquerda).

Seguindo o estudo de viabilidade $\mathrm{FIM}^{8}$ e a série de estudos nos quais os stents com eluição de sirolimus foram comparados aos stents não-farmacológicos em lesões relativamente mais simples, vieram as evidências clínicas dos estudos realizados em lesões mais complexas, como SVELTE ${ }^{14}$ e SES-SMART ${ }^{15}$ (vasos pequenos), SICTO $^{16}$ (oclusões crônicas), TROPICAL ${ }^{17}$ (reestenose intra-stent), ARTS ${ }^{18}$ (cirurgia de revascularização do miocárdio vs. angioplastia no paciente multiarterial), e DIABETES $^{19}$ (primeiro estudo com stent farmacológico realizado em uma população exclusivamente de pacientes diabéticos).

Desde que Kastrati et al. ${ }^{20}$ apresentaram os resultados provisórios do estudo ISAR-DESIRE, no ESC 2004, foi possível avaliar os dois stents com eluição de fármacos (sirolimus e paclitaxel) comercialmente disponíveis em cinco estudos comparativos.

O ISAR-DESIRE ${ }^{20}$ foi o primeiro estudo randomizado que demonstrou a superioridade dos stents com eluição de fármacos, quando comparados à angioplastia, no tratamento da reestenose intra-stent. Análises secundárias demonstram que pode haver superioridade do sirolimus sobre o paclitaxel, em termos de revascularização do vaso-alvo.

Resultados do SIRTAX ${ }^{21}$, envolvendo 1.012 pacientes, demonstraram que, quando comparado ao TAXUS, o CYPHER teve significativas menores taxas de morte, infarto agudo do miocárdio ou revascularização da lesão-alvo aos nove meses - desfecho composto primário $(6,2 \%$ vs. $10,8 \%$; $p<0,009)$. O mais expressivo desses achados é o fato de se tratar de estudo envolvendo uma população de pacientes complexos, em que foram incluídas oclusões crônicas, bifurcações, casos de síndrome coronariana aguda, enxertos de safena e lesões ostiais.

Seguindo a tendência determinada pelas comparações iniciais entre os stents com liberação de fármacos, os pacientes diabéticos incluídos no estudo ISAR-DI$\mathrm{ABETES}^{22}$ revelaram superioridade significativa do stent CYPHER quanto à perda luminal tardia e à supressão da hiperplasia neointimal, tanto intra-stent como no segmento da lesão. Esses achados correspondem a significativa redução das taxas de reestenose angiográfica.

Em comparação ao ISAR-DIABETES ${ }^{22}$ e ao SIRTAX ${ }^{21}$, a população do estudo REALITY ${ }^{23}$ pode ser descrita como de moderada complexidade. Apesar de não terem sido significativos, os achados do seguimento angiográfico de oito meses revelaram menores taxas de reestenose intra-stent e no segmento em favor do stent com eluição de sirolimus.

Dados adicionais do estudo SIRIUS ${ }^{10}$ servem para confirmar a durabilidade dos desfechos com o stent CYPHER e a importância em inibir a hiperplasia neointimal com a menor perda tardia. Os resultados do seguimento do estudo SIRIUS ${ }^{10}$, apresentados no American College of Cardiology (ACC), demonstraram diferenças significativas $(p<0,0001)$ entre o CYPHER e o stent não-farmacológico em todos os desfechos clínicos, que se mantiveram por três anos. Além disso, quando esses resultados são comparados aos dados do seguimento de nove meses, o SIRIUS ${ }^{10}$ demonstra que o benefício clínico do stent CYPHER em relação ao stent não-farmacológico continua crescendo.

Mais detalhes do CYPHER em lesões complexas provêm do estudo DANISH.

O SCANDSTENT ${ }^{24}$ é um estudo multicêntrico, randomizado, com 322 pacientes portadores de lesões complexas, tratadas tanto com stent CYPHER como com o stent não-farmacológico. Os resultados do seguimento de 12 meses do estudo ARTS II ${ }^{25}$ ampliaram as crescentes evidências, que dão respaldo ao uso de stents com eluição de sirolimus no tratamento de pacientes multiarteriais. Todavia, com tantas novas tecnologias, é necessário que se continue com pesquisas cuidadosas, que serão essenciais em auxiliar na escolha do paciente ideal e seu tratamento, envolvendo lesões mais complexas e pacientes multiarteriais (Figura 1).

Os casos de bifurcação do estudo CORPAL ${ }^{26}$, apresentados nas sessões científicas da American Heart Association (AHA) de 2005, compararam o stent com eluição de sirolimus e paclitaxel em uma variedade de pacientes portadores de lesões complexas. Lesões envolvendo bifurcações são complexas e, no geral, tecnicamente difíceis de tratar. Todos os pacientes incluídos nesse subgrupo tinham vasos com diâmetro $>2,5 \mathrm{~mm}$ e estenose significativa, tanto no vaso principal como em seu ramo lateral. Dados da angiografia 
quantitativa demonstraram, no seguimento de seis a nove meses, perda tardia significativamente menor com o CYPHER no vaso principal $(0,31 \mathrm{~mm}$ vs. $0,60 \mathrm{~mm}$; p < 0,05), assim como melhores desfechos clínicos em seis meses, relacionados à reestenose e à revascularização da lesão-alvo (Tabela 3).
Perda tardia: o ponto-chave na diferenciação dos stents com eluição de fármacos

A perda tardia é a medida angiográfica que permite quantificar de forma mais acurada e confiável o grau de hiperplasia neointimal após o implante de stents. Ela reflete a capacidade dos stents com eluição

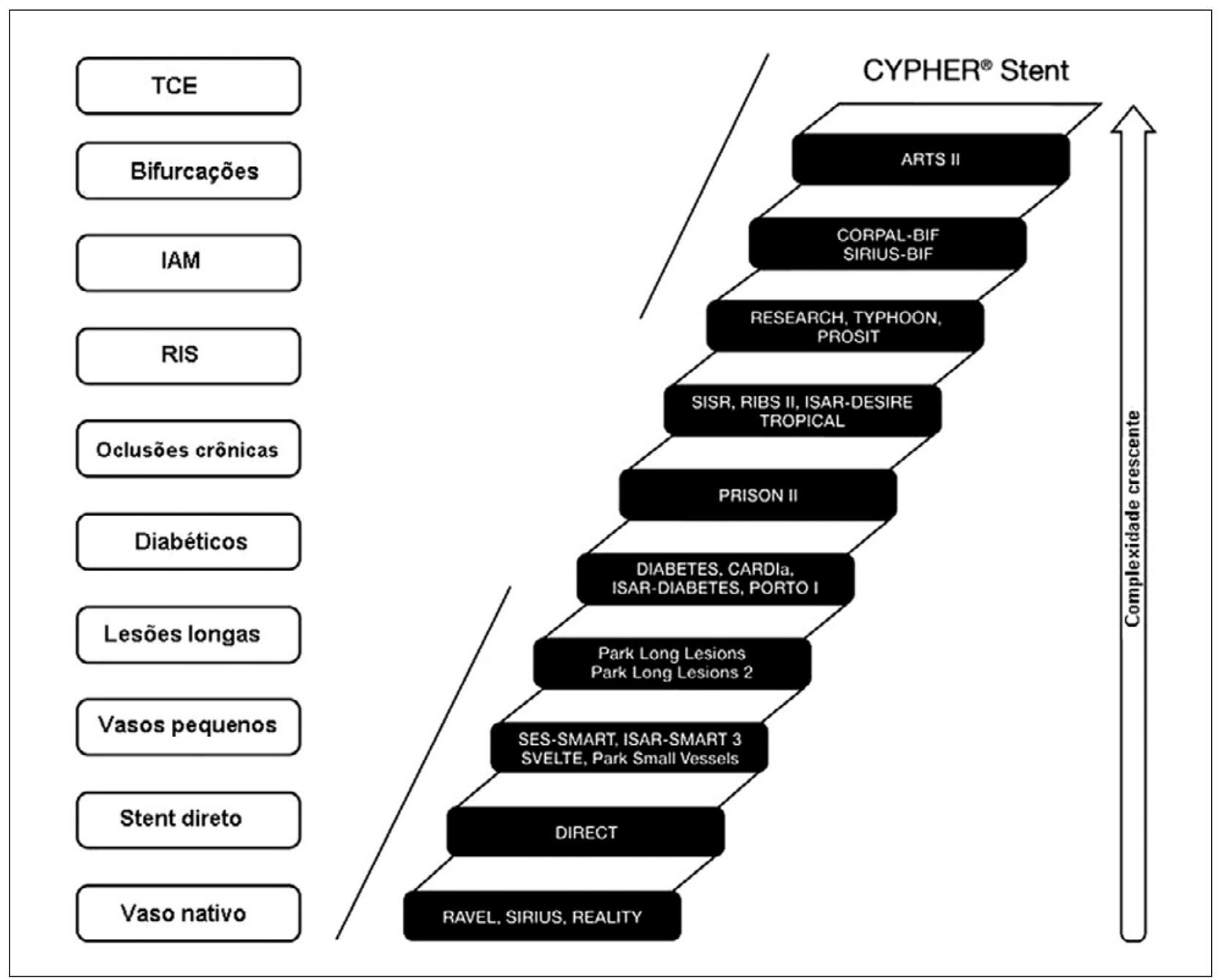

Figura 1 - Complexidade crescente dos estudos com sirolimus. TCE = tronco de coronária esquerda; IAM = infarto agudo do miocárdio; RIS $=$ reestenose intra-stent.

TABELA 3

Desfechos clínicos em seis meses do subgrupo de pacientes com lesões em bifurcações, incluídos no estudo CORPAL ${ }^{26}$

\begin{tabular}{lccc}
\hline Desfechos & CYPHER & TAXUS & p \\
\hline Morte & $1,3 \%$ & $1,3 \%$ & 1,0 \\
Infarto com onda Q & 0 & 0 & $\mathrm{~ns}$ \\
Infarto sem onda Q & 0 & $9,3 \%$ & 0,1 \\
Revascularização da lesão-alvo & $7,1 \%$ & $44,2 \%$ & $<0,0001$ \\
Eventos cardiovasculares maiores & $9,5 \%$ & $46,5 \%$ & $<0,0001$ \\
\hline
\end{tabular}


de fármacos em inibir o processo inflamatório e a hiperplasia neointimal, o que se reflete em desfechos clínicos adversos, como a reestenose binária e a revascularização da lesão-alvo.

Cinco estudos controlados, randomizados "cabeça a cabeça", comparando o TAXUS ao CYPHER, foram apresentados em 2004/2005: ISAR-DESIRE ${ }^{20}$, TAXI ${ }^{27}$, REALITY $^{23}$, SIRTAX ${ }^{21}$ e ISAR-DIABETES ${ }^{22}$. Esses estudos demonstraram vantagens consistentes e estatisticamente significantes com o uso do CYPHER no que se refere à perda tardia. Uma metanálise desses dados, apresentada no AHA de 2005, demonstrou que, entre todas as populações, o CYPHER teve menor perda tardia intra-stent que o TAXUS $(0,1 \mathrm{~mm}$ vs. $0,3 \mathrm{~mm})$, menores taxas de reestenose binária $(9,0 \%$ vs. $12,4 \% ; p=0,003)$ e revascularização da lesão-alvo $(0,5 \%$ vs. $12,5 \%$; $\mathrm{p}<0,01)$. Dados recentes do ENDEAVOR $\mathrm{II}^{28}$ e do CORPAL ${ }^{26}$ também demonstraram menor perda tardia com desfechos clínicos superiores nos pacientes que receberam o stent CYPHER.

Quando os estudos que compararam o CYPHER com o TAXUS são organizados de acordo com a crescente complexidade das lesões e dos pacientes (REALITY $\rightarrow$ SIRTAX $\rightarrow$ ISAR-DIABETES $\rightarrow$ ISAR-DESIRE), surge uma tendência indicando associação entre menor perda tardia e melhores desfechos clínicos (reestenose e revascularização da lesão-alvo). Quando a população de pacientes se torna mais complexa, a diferença entre o CYPHER e o TAXUS se amplia em termos de perda tardia e reestenose, demonstrando o benefício crescente do CYPHER (Figura 2).

Tem sido demonstrada associação entre perda tardia, reestenose e complexidade nos estudos com CYPHER vs. TAXUS. A perda tardia e a reestenose intra-stent aumentam quando a população de pacientes se torna mais complexa, sendo essa elevação mais consistente nos pacientes tratados com TAXUS. Essa tendência é expressa como curva de complexidade. Quando os resultados da perda tardia do ENDEAVOR III ${ }^{28}$ são expostos de acordo com a curva de complexidade, pode-se perceber que a perda tardia e as taxas de reestenose são consideradas altas em relação à relativa simplicidade dos pacientes incluídos nesse estudo (Figura 3).

\section{Design de células fechadas}

A distribuição do fármaco no tecido da parede do vaso não é totalmente homogênea, o que pode ser conseqüência do padrão das hastes do stent. A distribuição da dose pode ser subterapêutica em um local e tóxica em suas proximidades. A distribuição homogênea da droga também requer implante simétrico do stent, o que não necessariamente ocorre no mundo real. A sobreposição de stents pode levar ao dobro da dose prevista, levando à variação dos níveis da droga nos tecidos. O design do stent de células abertas apresenta diferentes características no padrão de aposição à parede do vaso, quando comparado ao do stent de células fechadas, levando a diferenças na dose efetiva, o que favorece este último por oferecer melhor distribuição da droga.

O stent CYPHER de células fechadas é uma plataforma ótima para liberação da droga, por apresentar cobertura uniforme do vaso. Esse design, quando implantado em local tortuoso, apresenta mínima alteração do tamanho das células, mantendo a homogeneidade tanto na cobertura do vaso como na liberação da droga.

No entanto, o stent de células abertas pode apresentar drástica mudança do tamanho de suas células, quando usado em vasos tortuosos, resultando em células muito abertas na curvatura maior e células muito fechadas na curvatura menor. Como conseqüência, não há cobertura uniforme da parede do vaso, levando à liberação irregular da droga.

\section{Polímero}

Apesar da distribuição e da segurança do fármaco, é necessário considerar sua relação com o design do stent e sua concentração nos tecidos. Os polímeros atualmente usados nos stents com eluição de fármacos apresentam segurança comprovada, liberando a droga de forma previsível e, surpreendentemente, com concentração tissular semelhante na forma de liberação lenta, quando comparada à de liberação rápida. A pe-

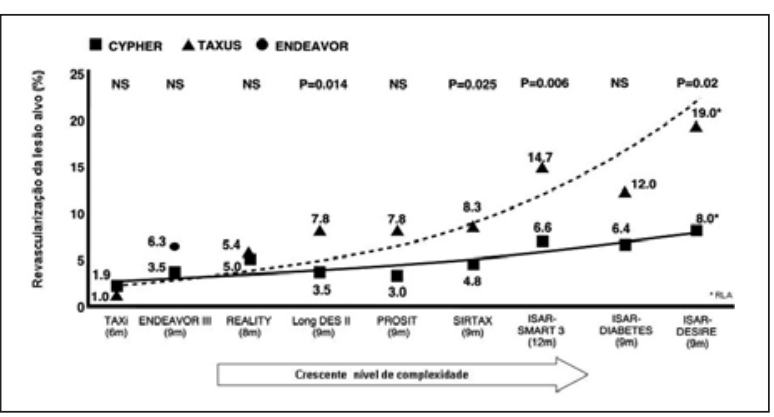

Figura 2 - Crescentes níveis de complexidade - Revascularização da lesão-alvo.

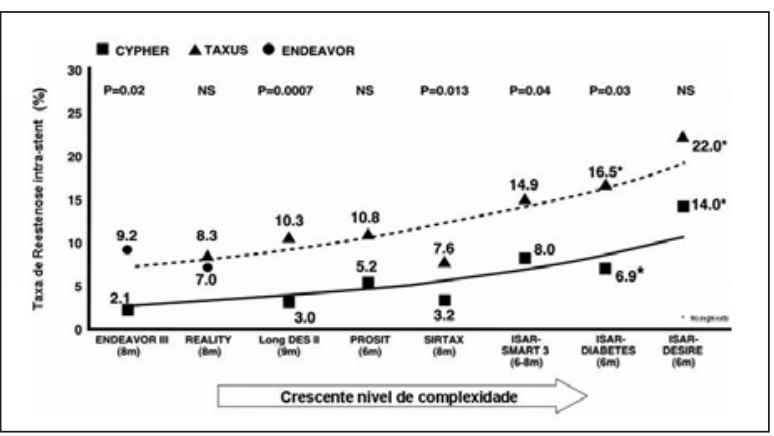

Figura 3 - Crescentes níveis de complexidade - Reestenose intra-stent. 
netração nos tecidos depende mais das propriedades lipofílicas da droga. Uma droga lipofílica (hidrofóbica) penetra facilmente nos tecidos, resultando em alta concentração, independentemente de sua liberação ser rápida ou lenta. A diferença na velocidade de liberação da droga pode ser responsável pela toxicidade tissular. A rápida liberação da droga com alta concentração nos tecidos pode ter efeitos tóxicos, levando à necrose do vaso. Isso pode formar trombos ou impossibilitar a aderência do stent à parede do vaso por tempo indeterminado, demonstrando a grande importância da toxicidade tanto clínica como histológica.

A liberação controlada da droga é crucial na eficácia dos stents com eluição de fármacos. O CYPHER é revestido por um polímero que permite a liberação localizada e precisa da droga no sítio da lesão. Esse stent apresenta concentração específica de sirolimus e seu polímero garante a permanência da droga, inclusive nos procedimentos de maior complexidade. O polímero também garante a liberação programada e definida em curto período de tempo. Em estudos experimentais, $100 \%$ da droga são liberados nos primeiros três meses após o implante do stent.

\section{Trombose tardia}

A trombose tardia é um evento adverso grave, definido como a oclusão do stent após os primeiros 30 dias do procedimento, sendo descrita, inicialmente, nos pacientes submetidos a braquiterapia intracoronária.

Grandes trabalhos randomizados comprovaram a expressiva superioridade dos stents com eluição de fármacos em reduzir as taxas de reestenose e a necessidade de nova intervenção. No entanto, o foco desses estudos não foi avaliar a incidência de eventos adversos menos freqüentes, como morte e trombose tardia.

Em março de 2006, a apresentação dos resultados do estudo BASKET-LATE ${ }^{29}$ sugeriu que, entre 7 e 18 meses, as taxas de infarto não-fatal, morte de causa cardíaca e trombose angiográfica do stent foram superiores com o uso de stents com eluição de fármacos, quando comparados aos stents não-farmacológicos.

A polêmica aumentou após os resultados das metanálises de stents com liberação de fármacos, apresentados no Congresso Mundial de Cardiologia de 2006, realizado em Barcelona. Esses estudos atribuíram aos stents farmacológicos maiores taxas de mortalidade no seguimento a longo prazo, emergindo, então, a necessidade de esclarecimento em relação aos dados já disponíveis e de realização de novos estudos com grande amostragem e desfecho primário relativo à segurança das próteses.

Lagerqvist et al. ${ }^{30}$ avaliaram 6.033 pacientes tratados com stents com eluição de fármacos (72\% TAXUS e 28\% CYPHER) e 13.738 pacientes com stents nãofarmacológicos por um período superior a três anos. Após ajuste pelas diferenças nas características basais, os autores concluíram que os stents com eluição de fármacos demonstraram aumento significativo dos casos de morte após seis meses do procedimento (risco relativo, 1,32; intervalo de confiança de 95\%, 1,11 a 1,57), com separação contínua das curvas de eventos (Figura 4).

Evento composto de morte ou infarto do miocárdio também foi significativamente desfavorável para o uso de stents com eluição de fármacos (risco relativo, 1,20; intervalo de confiança de 95\%, 1,05 a 1,37), após seis meses do procedimento (Figura 5).

Os resultados não demonstraram diferenças significativas em desfechos precoces $(p=0,40)$ ou tardios $(p=0,30)$ entre os pacientes que receberam stents com eluição de paclitaxel ou sirolimus.

Kastrati et al. ${ }^{31}$ fizeram uma metanálise com 4.958 pacientes incluídos em 14 estudos randomizados que compararam o stent com eluição de sirolimus aos sem eluição de fármacos. Os autores concluíram não haver diferença significativa quanto à mortalidade ou ao infarto do miocárdio, durante o seguimento de cinco anos.

Stone et al. ${ }^{32}$ analisaram os dados dos estudos RAVEL $^{9}$, SIRIUS ${ }^{10}$, E-SIRIUS ${ }^{11}$, C-SIRIUS $^{12}$ e a série $\operatorname{TAXUS}^{33}(\mathrm{I}, \mathrm{II}, \mathrm{IV}, \mathrm{V}$ e VI) e não observaram diferença

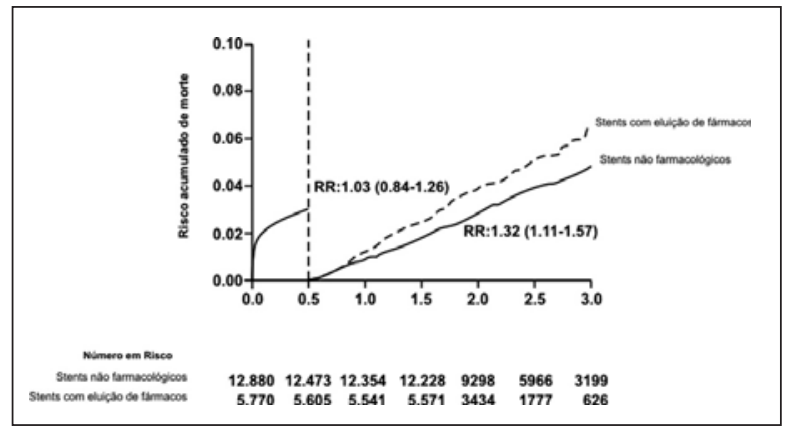

Figura 4 - Risco acumulado de morte (modificado de Lagerqvist et al. ${ }^{30}$ ). $\mathrm{RR}=$ risco relativo.

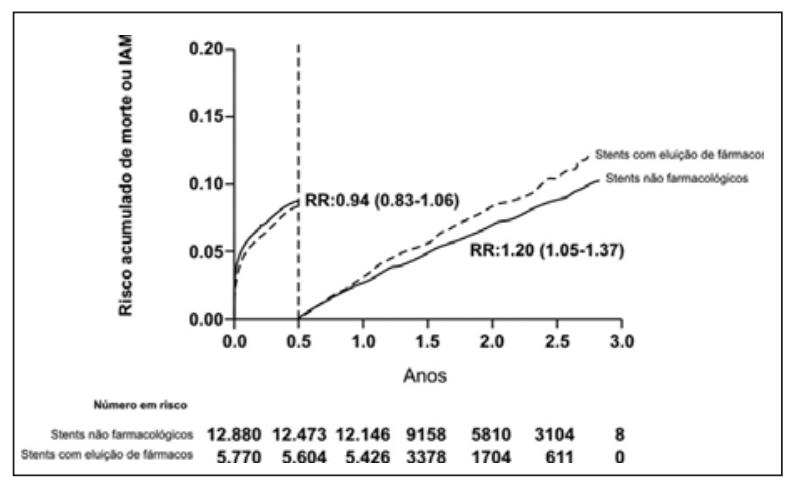

Figura 5 - Risco acumulado de morte ou infarto do miocárdio $\left(\right.$ modificado de Lagerqvist et $\left.\mathrm{al} .{ }^{30}\right) . \mathrm{RR}=$ risco relativo; $\mathrm{IAM}=$ infarto agudo do miocárdio. 
significativa nos índices de morte ou infarto entre os stents com eluição de fármacos e o grupo controle ( $p=0,20$ com sirolimus; $p=0,30$ com paclitaxel), no seguimento de quatro anos. Concluíram, ainda, haver aumento significativo de trombose dos stents com eluição de fármacos quando avaliados apenas após o primeiro ano do procedimento ( $p=0,025$ com sirolimus; $\mathrm{p}=0,028$ com paclitaxel).

Comparando o subgrupo de pacientes diabéticos desses mesmos estudos com sirolimus (RAVEL, SIRIUS, E-SIRIUS, C-SIRIUS) ${ }^{9-12}$, Spaulding et al. ${ }^{34}$ observaram significativa menor sobrevida dos pacientes que receberam o stent com eluição de fármaco, o que não foi demonstrado nos pacientes não-diabéticos (Figuras 6 e 7).

A taxa de trombose comprovada por angiografia foi de $4,4 \%$ no grupo sirolimus e de $0,8 \%$ no grupo controle, demonstrando que o diabetes é um preditor

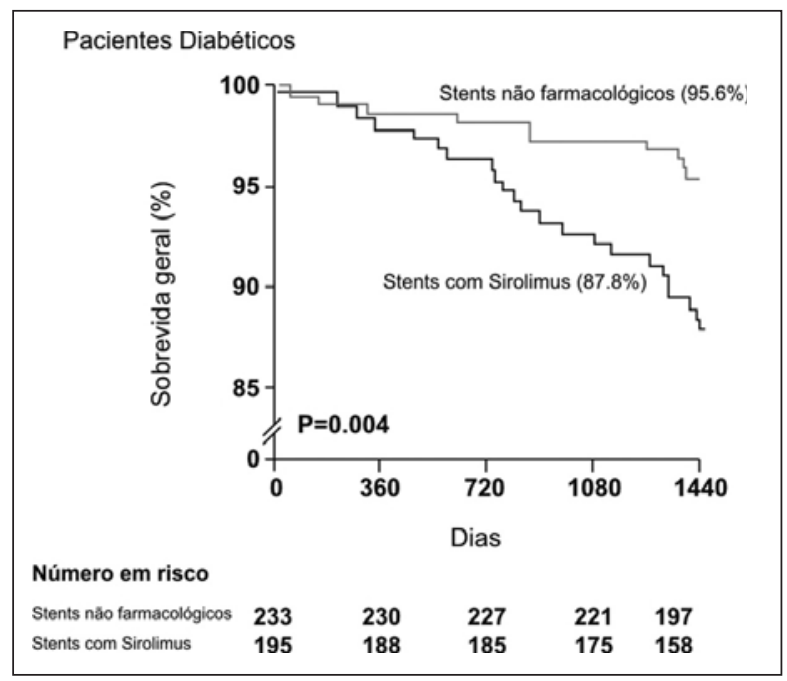

Figura 6 - Pacientes diabéticos (modificado de Spaulding et al. ${ }^{34}$ ).

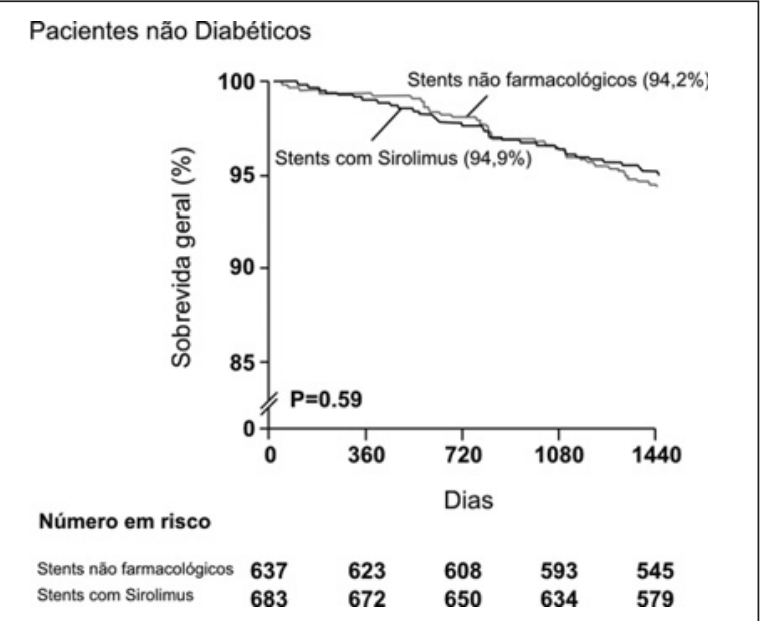

Figura 7 - Pacientes não-diabéticos (modificado de Spaulding et al. ${ }^{34}$ ). independente de trombose nos pacientes tratados com sirolimus.

Apesar de várias limitações desses estudos, como a pouca informação do tempo de tratamento com clopidogrel, o critério de seleção dos pacientes e as diferenças quanto a suas características basais, a trombose tardia é o maior temor da Cardiologia Intervencionista no momento. Nota-se maior índice de trombose tardia dos stents farmacológicos em um momento mais tardio quando comparado aos stents sem eluição de fármacos.

Dúvidas quanto ao tempo de manutenção da terapia antiplaquetária dupla permanecem motivo de grande controvérsia.

\section{Conclusões e situação atual}

A introdução do stent com eluição de fármacos foi a maior evolução da Cardiologia Intervencionista. Muitos estudos randomizados de grande porte demonstraram notória redução da reestenose angiográfica e da revascularização do vaso-alvo com esses stents, quando comparados aos stents não-farmacológicos. Os resultados desses estudos também são valorizados pelas evidências da prática diária e dos estudos não controlados. No entanto, a ampliação do uso de stents com liberação de sirolimus, especialmente no tratamento de lesões complexas, como em tronco de coronária esquerda, infarto agudo do miocárdio e enxertos de safena, ainda está sob investigação por estudos em andamento. O uso de stents com liberação de fármacos em todas as intervenções coronárias percutâneas pode se tornar realidade em futuro próximo.

Com o aprimoramento da interação entre plataforma dos stents, polímero e dose ideal do fármaco, poderá ser aumentado o espectro de segurança desses dispositivos para seu uso universal.

\section{REFERÊNCIAS BIBLIOGRÁFICAS}

1. Grüntzig A. Transluminal dilatation of coronary artery stenosis. Lancet. 1978;1(8058):263.

2. Costa MA, Simon DI. Molecular basis of restenosis and drug-eluting stents. Circulation. 2005;111(17):2257-73.

3. Hoffmann R, Mintz GS, Dussaillant GR, Popma JJ, Pichard $A D$, Satler LF, et al. Patterns and mechanisms of in-stents restenosis: a serial intravascular ultrasound study. Circulation. 1996;94(6):1247-54.

4. Sehgal SN, Baker H, Vezina C. Rapamycin (AY-22,989), a new antifungal antibiotic. II. Fermentation, isolation and characterization. J Antibiot. (Tokyo) 1975;28(10):727-32.

5. Singh K, Sun S, Vezina C. Rapamycin (AY-22,989), a new antifungal antibiotic. VI. Mechanism of action. J Antibiot (Tokyo). 1979;32(6):630-45.

6. Chang JY, Sehgal SN, Bansbach CC. FK506 and rapamycin: novel pharmacological probes of the immune response. Trends Pharmacol Sci. 1991;12(6):218-23.

7. Marx SO, Jayaraman T, Go LO, Marks AR. Rapamycin-FKBP inhibits cell cycle regulators of proliferation in vascular smooth muscle cells. Circ Res. 1995;76(3):412-7. 
8. Sousa JE, Costa MA, Abizaid A, Abizaid AS, Feres F, Pinto $I M$, et al. Lack of neointimal proliferation after implantation of sirolimus-coated stents in human coronary arteries: a quantitative coronary angiography and three-dimensional intravascular ultrasound study. Circulation. 2001;103(2):192-5.

9. Morice MC, Serruys PW, Sousa JE, Fajadet J, Ban Hayashi E, Perin M, et al. For the RAVEL Study Group. Randomized Study with the Sirolimus-Coated Bx Velocity Balloon-Expandable Stent in the Treatment of Patients with de Novo Native Coronary Artery Lesions. N Engl J Med. 2002;346(23): 1773-80.

10. Moses JW. A U.S multicenter, randomized, double-blind study of the sirolimus eluting stent in de novo native coronary lesions. In: Transcatheter Cardiovascular Therapeutics; 2002.

11. Schofer J, Schluter M, Gershlick AH, Wijns W, Garcia E, Schampaert E, et al. Sirolimus-eluting stents for treatment of patients with long atherosclerotic lesions in small coronary arteries: double-blind, randomized controlled trial (E-SIRIUS). Lancet. 2003;362(9390):1093-9.

12. Schampaert E, Cohen EA, Schluter M, Reeves F, Traboulsi M, Title LM, et al. The Canadian Study of the Sirolimuseluting Stent in the treatment of patients with long de novo lesions in small native coronary arteries (C-SIRIUS). J Am Coll Cardiol. 2004;43(6):1110-5.

13. Hirohata A, Morino Y, Ako J, Sakurai R, Buchbinder M, Caputo RP, et al. Comparison of the efficacy of direct coronary stenting with sirolimus-eluting stents versus stenting with predilation by intravascular ultrasound imaging (from the DIRECT trial). Am J Cardiol. 2006;98(11):1464-7.

14. Devito FS, Sousa AG, Feres F, Abizaid AA, Staico R, Mattos LA, et al. Comparative analysis of intimal hyperplasia after sirolimus-eluting stent and thin-strut bare-metal stent implantation in small coronary arteries. Arq Bras Cardiol. 2006;86(4):268-75.

15. Ortolani P, Ardissino D, Cavallini C, Bramucci E, Indolfi C, Aquilina $M$, et al. Effect of sirolimus-eluting stent in diabetic patients with small coronary arteries (a SES-SMART substudy). Am J Cardiol. 2005;96(10):1393-8.

16. Lotan C, Almagor $Y$, Kuiper K, Suttorp MJ, Wijns W. Sirolimuseluting stent in chronic total occlusion: the SICTO study. J Interv Cardiol. 2006;19(4):307-12.

17. Leon MB, Teirstein PS, Moses JW, Tripuraneni P, Lansky AJ, Jani $S$, et al. Localized intracoronary gamma-radiation therapy to inhibit the recurrence of restenosis after stenting. N Engl J Med. 2001;344(4):250-6.

18. Serruys PW, Unger F, Sousa JE, Jatene A, Bonnier HJ, Schönberger JP, et al. Comparison of coronary-artery bypass surgery and stenting for the treatment of multivessel disease. N Engl J Med. 2001;344(15):1117-24.

19. Sabate $M$, Jimenez-Quevedo P, Angiolillo DJ, Gomez-Hospital JA, Alfonso F, et al. Randomized comparison of sirolimuseluting stent versus standard stent for percutaneous coronary revascularization in diabetic patients: the diabetes and sirolimus-eluting stent (DIABETES) trial. Circulation. 2005;112 (14):2175-83.

20. Kastrati A, Mehilli J, von Beckerath N, Dibra A, Hausleiter J, Pache J, et al. Sirolimus-eluting stent or paclitaxel-eluting stent vs balloon angioplasty for prevention of recurrences in patients with coronary in-stent restenosis: a randomized controlled trial. JAMA. 2005;293(2):165-71.
21. Windecker $S$, Remondino $A$, Eberli $F R$, Jüni $P$, Räber L, Wenaweser $\mathrm{P}$, et al. Sirolimus-eluting and paclitaxel-eluting stents for coronary revascularization. N Engl J Med. 2005;353 (7):653-62

22. Dibra A, Kastrati A, Mehilli J, Pache J, Schühlen H, von Beckerath $\mathrm{N}$, et al. Paclitaxel-eluting or sirolimus-eluting stents to prevent restenosis in diabetic patients. N Engl J Med. 2005;353(7):663-70.

23. Morice MC, Colombo A, Meier B, Serruys P, Tamburino C, Guagliumi G, et al. Sirolimus- vs paclitaxel-eluting stents in de novo coronary artery lesions: the REALITY trial: a randomized controlled trial. JAMA. 2006;295(8):895-904.

24. Kelbaek H, Thuesen L, Helqvist S, Klovgaard L, Jorgensen E, Aljabbari S, et al. The Stenting Coronary Arteries in Nonstress/benestent Disease (SCANDSTENT) trial. J Am Coll Cardiol. 2006;47(2):449-55.

25. Serruys PW, Lemos PA, van Hout BA. Sirolimus eluting stent implantation for patients with multivessel disease: rationale for the Arterial Revascularisation Therapies Study part II (ARTS II). Arterial Revascularisation Therapies Study part II Steering Committee and Investigators. Heart. 2004;90 (9):995-8

26. Kastrati A, Dibra A, Eberle S, Mehilli J, Suarez de Lezo J, Goy J, et al. Sirolimus-eluting stents vs paclitaxel-eluting stents in patients with coronary artery disease: meta-analysis of randomized trials. JAMA. 2005;294(7):819-25.

27. Goy JJ, Stauffer JC, Siegenthaler M, Benoit A, Seydoux C. A prospective randomized comparison between paclitaxel and sirolimus stents in the real world of interventional cardiology: the TAXi trial. J Am Coll Cardiol. 2005;45(2):308-11.

28. Kandzari DE, Leon MB, Popma JJ, Fitzgerald PJ, O'Shaughnessy C, Ball MW, et al. Comparison of zotarolimus-eluting and sirolimus-eluting stents in patients with native coronary artery disease: a randomized controlled trial. J Am Coll Cardiol. 2006;48(12):2440-7.

29. Kaiser C, Brunner-La Rocca HP, Buser PT, Bonetti PO, Osswald S, Linka A, et al; BASKET Investigators. Incremental cost-effectiveness of drug-eluting stents compared with a third-generation bare-metal stent in a real-world setting: randomised Basel Stent Kosten Effektivitats Trial (BASKET). Lancet. 2005;366(9489):921-9.

30. Lagerqvist B, James SK, Stenestrand U, Lindback J, Nilsson $\mathrm{T}$, Wallentin L. Long-term outcomes with drug-eluting stents versus bare-metal stents in Sweden. N Engl J Med. 2007;356(10):1009-19.

31. Kastrati A, Mehilli J, Pache J, Kaiser C, Valgimigli M Kelbaek $\mathrm{H}$, et al. Analysis of 14 trials comparing sirolimuseluting stents with bare-metal stents. N Engl J Med. 2007;356(10):1030-9.

32. Stone GW, Moses JW, Ellis SG, Schofer J, Dawkins KD, Morice MC, et al. Safety and efficacy of sirolimus- and paclitaxel-eluting coronary stents. N Engl J Med. 2007;356(10): 998-1008.

33. Stone G. TAXUS I- VI: results and trials with a polymerbased paclitaxel delivery system (March 2002). Disponível em URL: http:/www.tctmd.com/expert-presentations/multi-slide.html?product_id=1972(2002/10/03).

34. Spaulding C, Daemen J, Boersma E, Cutlip DE, Serruys PW. A pooled analysis of data comparing sirolimus-eluting stents with bare-metal stents. N Engl J Med. 2007;356(10):989-97. 